SCIENTIFIC LETTER

\title{
Relative importance of the components of stent geometry to stretch induced in-stent neointima formation
}

\author{
C J Dean, A C Morton, N D Arnold, D R Hose, D C Crossman, J Gunn
}

Heart 2005;91:1603-1604. doi: 10.1136/hrt.2004.047050

$\mathrm{E}$ ven in the era of drug eluting stents, in-stent restenosis is likely to remain a significant problem. Restenosis is caused by growth of a neointima, comprised of smooth muscle cells and intercellular matrix. The quantity of neointima is directly proportional to the amount of injury inflicted upon the artery by the stent itself. ${ }^{1}$ That injury is in the form of either a deep laceration or stretch of the arterial wall. We have previously shown that stretch is ubiquitous after stenting and, even in the absence of deep injury, is an important mediator of neointima formation. ${ }^{2}$ A few parameters of stent geometry have been shown to have an influence upon neointima formation, including smaller strut number, ${ }^{3}$ greater strut thickness, ${ }^{4}$ and increased variation in the angular burden (pointedness) of the stent cross section.

Therefore, in this study, we aimed to examine the contribution of a wide panel of parameters of stent geometry to the development of neointima in stent sections that displayed stretch but not deep injury.

\section{METHODS}

In accordance with UK Home Office regulations, we deployed BiodivYsio stents $(3.5 \times 15 \mathrm{~mm})$ in 26 coronary arteries of 21 pigs; 17 in the right coronary artery (RCA) and nine in the left anterior descending (LAD). Quantitative coronary angiography identified two segments of artery where the balloon to artery ratio was $1.25: 1$. The deploying balloon was inflated to 8 atmospheres for 30 seconds; $2500 \mathrm{U}$ heparin was given before implantation and $150 \mathrm{mg}$ of aspirin was given orally for five days. At 28 days the animals were sacrificed and the stented artery segments flushed and immersion fixed in formalin, cut, ground, and polished into 16-20 histological sections. Imperfect sections and those with deep injury were excluded from the analysis. Neointimal area for each section, and neointimal thickness over each strut, was measured using computerised morphometry. Strut depth, strut width, inter-strut distance, protrusion of each strut from its neighbours, angular burden of the strut into the internal elastic lamina, medial bowing, and neointimal thickness was measured for each strut $(n=1000)$, with its associated neointimal thickness. For each section $(n=99)$, with its associated neointimal and luminal areas, we measured the number of struts, percentage metal coverage of the arterial wall, stent major and minor axes, stent oblateness, and areas of the lumen and the neointima. We also calculated the maximum, mean, and standard deviation of these parameters. All of these parameters are explained in fig 1.

Raw data are expressed as mean (SEM). Per strut statistics were analysed with ANOVA; this tested the significance of each independent parameter (strut depth, thickness and so on) against the dependent parameter, neointimal thickness. This test was performed with strut sample sizes of 1000, 250, and 50 to obtain a rank order of significance of the independent parameters. Per section statistics were analysed with stepwise linear regression analysis. Each independent parameter (stent geometry) was tested for significance against each dependent parameter (lumen and neointima areas and thicknesses) to yield a list of stent geometry parameters that correlated with arterial morphometry. The regression coefficient for each independent variable was calculated with its respective significance level.

\section{RESULTS}

Ninety nine stented artery sections containing 1000 struts were available for analysis. The number of strut elements in each cross section was 10.1 (0.3); strut depth was 0.149 $(0.001) \mathrm{mm}$; strut width was 0.349 (0.007) $\mathrm{mm}$; metal coverage was 40 (2)\%; inter-strut distance was 0.53 (0.01) $\mathrm{mm}$; strut protrusion was 0.293 (0.007) $\mathrm{mm}$; angular burden was 106.4 (0.9) degrees; medial bowing was 0.004 $(0.002) \mathrm{mm}$; and oblateness was 1.077 (0.007). The cross sectional area of the lumen was $4.5(0.1) \mathrm{mm}^{2}$ and of the neointima $1.34(0.08) \mathrm{mm}^{2}$. The thickness of the neointima at each strut was $0.17(0.01) \mathrm{mm}$.

Per strut analysis revealed five predictors of neointimal thickness. In order of significance, they were strut protrusion $(p<0.001)$, inter-strut distance $(p<0.001)$, medial bowing $(\mathrm{p}<0.001)$, strut depth $(\mathrm{p}<0.001)$, and angular burden $(\mathrm{p}<0.001)$. Strut thickness was not a significant predictor.

Per section analysis revealed that lumen area correlated negatively with mean medial bowing $(p<0.001)$ and

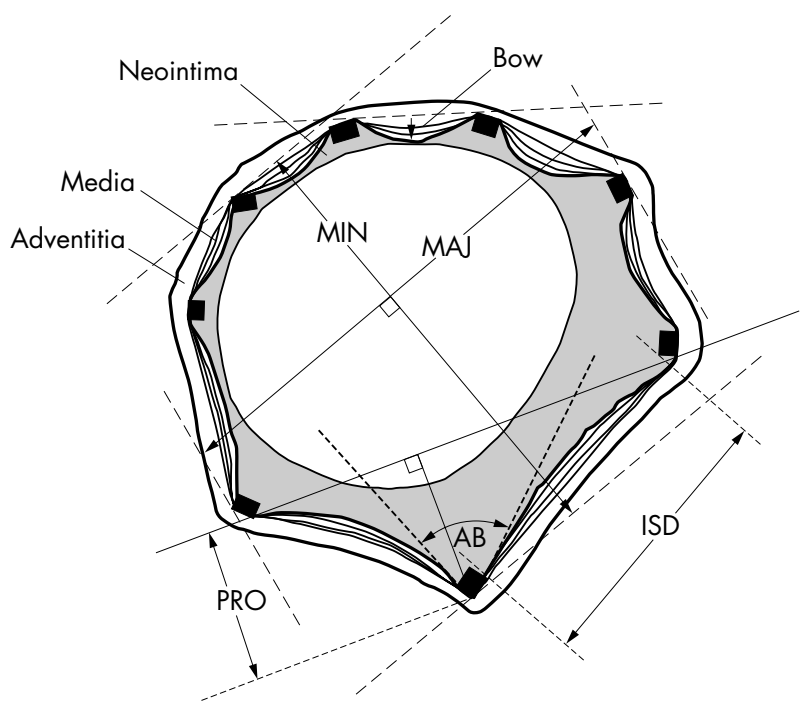

Figure 1 Diagram of a section of a stented artery. Ninety nine sections from stented porcine coronary arteries displaying stretch (but not deep injury) containing a total of 1000 struts were analysed. Highly significant associations were found, by analysis of variance (ANOVA), between neointimal thickness and, in rank order: strut protrusion, inter-strut distance (ISD), medial bowing, strut depth, angular burden (AB), and strut thickness. No association was found with the number of struts in the section or with the major or minor axis measurements. 
positively with mean angular burden $(p<0.001)$, major axis $(\mathrm{p}=0.002)$, minor axis $(\mathrm{p}=0.022)$, and maximum angular burden $(p=0.036)$. Neointimal area correlated positively with major axis $(\mathrm{p}<0.001)$, mean medial bowing $(\mathrm{p}<0.001)$, mean angular burden $(\mathrm{p}=0.001)$, and negatively with mean protrusion $(\mathrm{p}=0.021)$.

\section{DISCUSSION}

We have shown that stent induced stretch of coronary arteries results in a neointima whose magnitude is directly related to a number of simple parameters of stent geometry. In particular, neointimal thickness at a strut is related to strut protrusion, inter-strut distance, medial bowing, strut depth, and angular burden. Neointimal area is related to major axis, mean medial bowing, mean angular burden, and mean strut protrusion. While other authors have examined individual parameters, such as strut number, ${ }^{3}$ strut thickness, ${ }^{4}$ and angular burden, ${ }^{5}$ this is the first study to investigate all potential factors together, and to assign a relative importance to each.

It has been suggested that stent design is unimportant to restenosis. We have shown this to be incorrect. While novel stent designs are directed by industrial ingenuity towards enhancing deliverability, conformability, and radial strength (all highly desirable attributes), control of the biological response may also be possible through careful manipulation of stent design, to enhance the beneficial effect of stent coatings and drugs.

\section{ACKNOWLEDGEMENTS}

We are grateful to the Special Trustees of the former United Sheffield Hospitals' Charitable Funds and to Abbott Vascular
Devices for their support of this study and to Heather Allen for the illustration.

\section{Authors' affiliations}

C J Dean, D R Hose, Department of Medical Physics and Clinical Engineering, University of Sheffield, Sheffield, UK

A C Morton, N D Arnold, D C Crossman, J Gunn, Cardiovascular Research Unit, Division of Clinical Sciences, Northern General Hospital, University of Sheffield

Conflict of interest: Dr Gunn has performed paid consultancy work for Abbott Vascular Devices and Biocompatibles (current and previous manufacturers of the BiodivYsio stent). Those companies have also supported several of his research projects.

Correspondence to: Dr Julian Gunn, Cardiovascular Research Unit, Clinical Sciences Building, Northern General Hospital, Sheffield, S5 7AU, UK; i.gunn@sheffield.ac.uk

Accepted 15 March 2005

\section{REFERENCES}

1 Schwartz RS, Huber KC, Murphy JG, et al. Restenosis and the proportional neointimal response to coronary artery injury: results in a porcine model. J Am Coll Cardiol 1992;19:267-74.

2 Gunn J, Arnold N, Chan KH, et al. Coronary artery stretch versus deep injury in the development of in-stent neointima. Heart 2002;88:401-5.

3 Garasic JM, Edelman ER, Squire JC, et al. Stent and artery geometry to determine intimal thickening independent of arterial injury. Circulation 2000;101:812-8.

4 Kastrati A, Stühlen $\mathrm{H}$, Hausleiter J, et al. Intracoronary stenting and angiographic results: strut thickness effect on restenosis outcome (ISARSTEREO) trial. Circulation 2001;103:2816-21.

5 Schultz C, Herrmann RA, Beilharz C, et al. Coronary stent symmetry and vascular injury determine experimental restenosis. Heart 2000;83:462-7.

\title{
IMAGES IN CARDIOLOGY
}

\section{The invisible stent: imaging of an absorbable metal stent with multislice spiral computed tomography}

\begin{abstract}
A 60 year old man was admitted for recurrent stress dependant episodes of chest pain radiating toward the left shoulder for three months. Laboratory tests showed normal troponin I concentrations. During exercise testing the ECG documented significant ST segment depression $>1 \mathrm{~mm}$ in V1-V3. Informed consent was obtained and coronary angiography was performed. Examination revealed a single segmental stenosis of the mid left anterior descending coronary artery (LAD) (segment 7, 65\% diameter stenosis).

Successful placement of a single absorbable metal stent (AMS) (Biotronik, Bulach, Switzerland) was accomplished in the catheterisation laboratory under fluoroscopic and intravascular ultrasound (IVUS) guidance.

After stent placement a contrast enhanced multislice spiral computed tomography (MSCT) (Somatom Sensation 16, Siemens, Forchheim, Germany) scan was performed using a $500 \mathrm{~ms}$ rotation time and $0.75 \mathrm{~mm}$ slice thickness during a 30 second breathhold. MSCT demonstrated adequate perfusion throughout the entire LAD without signs of stenosis in the stented area (panel B).
\end{abstract}
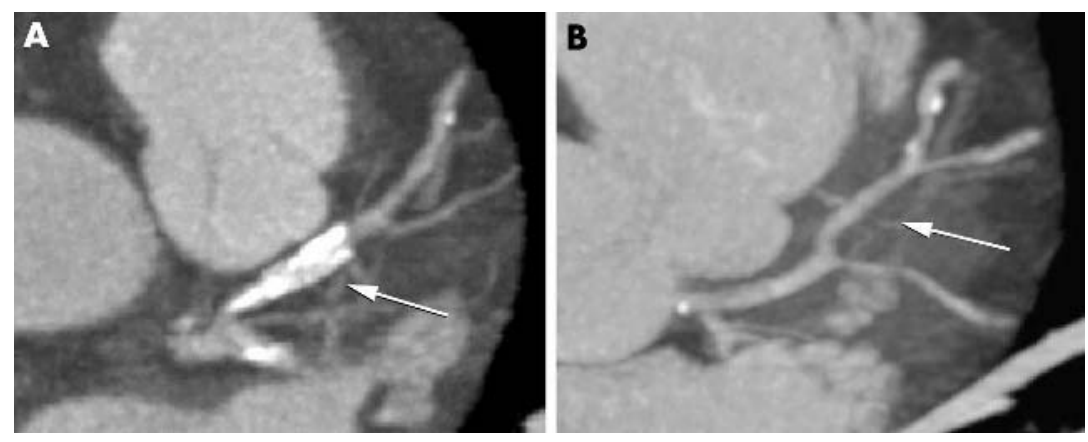

The AMS therefore overcomes the imaging problems of conventional metallic stents (panel A) and enables reliable direct visualisation of coronary arteries. Thus, non-invasive follow up with MSCT of patients treated with AMS might be feasible for the first time because of adequate differentiation between stent patency, in-stent stenosis, and stent occlusion.

A Y Lind

H Eggebrecht

R Erbel

lind@gmx.org 